\author{
Marella F. T. R. de Bruijn $\triangle$, \\ Walentina A. T. Slieker ${ }^{\triangle}$, \\ Johannes C. M. van der Loo ${ }^{\bullet}$, \\ Jane S. A. Voerman $\triangle$, \\ Willem van Ewijk ${ }^{\triangle}$ and \\ Pieter J. M. Leenen ${ }^{\wedge}$
}

Department of Immunology ${ }^{\triangle}$ and Department of Hematology ${ }^{\bullet}$, Erasmus University, Rotterdam

\section{Distinct mouse bone marrow macrophage precursors identified by differential expression of ER-MP12 and ER-MP20 antigens}

The characterization of early branch points in the differentiation of leukocytes requires identification of precursor cells in the bone marrow. Recently, we produced two monoclonal antibodies, ER-MP12 and ER-MP20, which in two-color flow-cytometric analysis divide the murine bone marrow into six defined subsets. Here we show, using fluorescence-activated cell sorting followed by macrophage colony-stimulating factor-stimulated culture in soft agar, that precursors of the mononuclear phagocyte system reside only within the ER-MP12 ${ }^{\text {hi }} 20^{-}$, ER-MP12 $20^{+}$and ER-MP12- $20^{\text {hi }}$ bone marrow subsets. Together, these subsets comprise $15 \%$ of nucleated bone marrow cells. Furthermore, we provide evidence that the macrophage precursors present in these subsets represent successive stages in a maturation sequence where the most immature ER-MP12 $22^{\text {hi }} 20^{-}$cells develop via the ER-MP12+ $20^{+}$stage into ER-MP12-20 ${ }^{\text {hi }}$ monocytes.

\section{Introduction}

Macrophages form a heterogeneous population of cells which play essential roles in a wide variety of biological processes (for reviews see $[1,2]$ ). As yet, it is not clear whether their extensive diversity is generated solely at the level of the monocytes entering the tissue microenvironments, or also at the level of the macrophage precursors in the bone marrow. Studies on the early stages of macrophage development have been seriously hampered by the limited characterization and low frequency of macrophage precursors in the bone marrow. To approach this problem, we previously produced a panel of monoclonal antibodies (mAb) using immortalized macrophage precursors as immunogens [3]. Two of these mAb, ER-MP12 and ERMP20, were shown to detect phenotypic heterogeneity among bone marrow macrophage precursors [3]. In the present study we aimed at identifying distinct macrophage precursor subsets in mouse bone marrow using ER-MP12 and ER-MP20 mAb in two-color flow-cytometric analysis and cell sorting. Bone marrow subsets sorted on the basis of their differential expression of ER-MP12 and ER-MP20 antigens were examined on cellular composition, macrophage colony/cluster-forming capacity, cell surface expression of the macrophage maturation-related marker Mac-1, and expression of ER-MP12 and ER-MP20 antigens during macrophage maturation in vitro. In this report we show that three phenotypically distinct subsets of M-CSF-responsive bone marrow macrophage precursors can be identified. Moreover, our data indicate that these three macrophage precursor subsets reflect successive, phenotypically defined, stages of in vivo macrophage development.

\section{[I 13171]}

Correspondence: Marella de Bruijn, Department of Immunology, Erasmus University, P.O. Box 1738, NL-3000 DR Rotterdam, The Netherlands (Fax: 31-10-4367601)

Abbreviations: M-CSF: Macrophage colony-stimulating factor M-CFC: Macrophage colony-forming cell

Key words: Macrophage / Progenitor / Heterogeneity / Phenotype

\section{Materials and methods}

\subsection{Mice}

Female C57BL/6-Ly-5.1-Pep ${ }^{3 b}$ mice (breeding pairs kindly provided by Dr. I. L. Weissman, Stanford University, Stanford, CA) between 6 and 20 weeks old were used in all experiments. Animals were kept under clean routine laboratory conditions with free access to food and water.

\subsection{Antibodies and conjugates}

The mAb used in this study were M1/70 (anti-Mac-1 [4]), ER-BMDM1 (anti-aminopeptidase N [5]), ER-MP12 [3], and ER-MP20 (anti-Ly-6C [3]). Antibodies were applied as hybridoma culture supernatants or as purified $m A b$ conjugated to FITC (fluorescein isothiocyanate, isomer I, Sigma Chemical Co., St. Louis, MO) or biotin (N-hydroxysuccinimidobiotin, Boehringer Mannheim GMBH, Mannheim, FRG) by standard procedures.

R-Phycoerythrin-conjugated streptavidin (SAV-PE; Caltag Laboratories, CA), Tri-Color-conjugated streptavidin (SAV-TC; Caltag Laboratories), FITC-conjugated rabbit anti-rat IgG F(ab) ${ }_{2}$ fragments (R $\alpha$ Ra-FITC; Cappel, Organon Teknika, Turnhout, Belgium), and R-phycoerythrinconjugated goat-anti-rat IgG (mouse-adsorbed; GaRa-PE; Caltag Laboratories), were used as second stage fluorescent reagents.

\subsection{Preparation of cell suspensions}

Bone marrow cell suspensions were prepared as described previously [6]. Briefly, femora and tibiae were ground, using a mortar, in Dutton's Balanced Salt Solution (Gibco, Breda, The Netherlands) supplemented with $5 \%$ fetal calf serum, $60 \mu \mathrm{g} / \mathrm{ml}$ penicillin and $100 \mu \mathrm{g} / \mathrm{ml}$ streptomycin (DBSS-FCS-PS). The cell suspension was aspirated through a 22-gauge needle and filtered over a nylon sieve (mesh size $100 \mu \mathrm{m}$; Polymon PES, Kabel, Amsterdam, The Netherlands) to remove connective tissue, bone fragments, and clumps of cells. 
Cultured bone marrow cells were isolated from Teflon bags (see below) and washed with DBSS-FCS-PS prior to immunofluorescence labeling.

\subsection{Immunofluorescence labeling, flow-cytometric analysis and cell sorting}

For phenotypic analyses, $1 \times 10^{6}-5 \times 10^{6}$ freshly isolated bone marrow cells/well or $5 \times 10^{4}$ cultured cells/well were aliquotted into 96-microwell plates (round-bottom, Nunc, Denmark) and labeled with the appropriate antibodies in phosphate-buffered saline supplemented with $0.5 \%$ bovine serum albumin and $20 \mathrm{mM} \mathrm{NaN}_{3}$ (PBS-BSA-NaN $\mathrm{Na}_{3}$ ). All incubations were performed on ice for $30 \mathrm{~min}$ and were followed by three washes with PBS-BSA-NaN 3 . For singlecolor analysis, cells were incubated first with hybridoma supernatant, washed and then incubated with RoRa-FITC, supplemented with $2 \%$ normal mouse serum (NMS) to avoid nonspecific binding. For two-color analysis, cells were incubated first with biotinylated $\mathrm{mAb}$, followed by FITClabeled $\mathrm{mAb}$ and SAV-PE simultaneously. For three-color analysis, cells were incubated first with hybridoma supernatant followed by GaRa-PE. After two washes, cells were washed in the presence of $3 \%$ normal rat serum to block free binding sites on GaRa-PE. Subsequently, cells were incubated with biotinylated $\mathrm{mAb}$ followed by FITClabeled $\mathrm{mAb}$ and SAV-TC simultaneously. Percentages of positive cells obtained from three-color analysis were compared with those obtained from single stainings and were found to be identical (data not shown). Culture supernatant of the nonproducing Y3 myeloma followed by $\mathrm{R} \alpha \mathrm{Ra}$-FITC (one-color analysis) or GaRa-PE (three-color analysis) was used as negative control, since, in our hands, control values obtained with Y3 supernatant are identical to those obtained with rat isotype control $\mathrm{mAb}$ (unpublished data). Phenotypic analyses were performed with a FACScan cytofluorimeter (Becton Dickinson, Sunnyvale, CA).

For cell sorting experiments, $2 \times 10^{8}$ bone marrow cells were incubated for $30 \mathrm{~min}$ with $2 \mathrm{ml}$ DBSS-FCS-PS containing biotinylated ER-MP12, washed with a large volume of DBSS-FCS-PS and subsequently incubated (30 min) with $2 \mathrm{ml}$ optimally diluted ER-MP20-FITC and SAV-PE simultaneously. After two washes, the cells were resuspended in PBS supplemented with BSA $(0.5 \% \mathrm{w} / \mathrm{v})$, D-glucose $(0.45 \%$ w/v; Merck, Amsterdam, The Netherlands), penicillin $(60 \mu \mathrm{g} / \mathrm{ml})$ and streptomycin $(100 \mu \mathrm{g} / \mathrm{ml})$ to a final concentration of $3 \times 10^{6}$ cells $/ \mathrm{ml}$. Before sorting, the cell suspension was filtered over a $30-\mu \mathrm{m}$ sieve (Polymon PES) to avoid clogging of the nozzle. All sorts were performed using a FACS Vantage cell sorter (Becton Dickinson). After sorting, viable cells were counted using a Bürker hemocytometer. The purity of the sorted cell populations was determined by FACScan analysis and exceeded $95 \%$, unless stated otherwise.

\subsection{M-CSF-stimulated bone marrow culture}

The medium used for M-CSF-stimulated bone marrow culture (either in soft agar or in Teflon culture bags, see below) was $\alpha$-modified DMEM (Gibco) supplemented with $20 \% \mathrm{~L}$ cell-conditioned medium (LCM) as a source of M-CSF, $20 \%$ FCS (heat-inactivated; Hyclone Laborato- ries, UT), glutamine (2 $\mathrm{mM})$, penicillin $(60 \mu \mathrm{g} / \mathrm{ml})$, streptomycin $(100 \mu \mathrm{g} / \mathrm{ml}), \beta$-mercaptoethanol $\left(10^{-4} \mathrm{M}\right)$ and sodium selenite $\left(10^{-7} \mathrm{M}\right)$. LCM was prepared as previously described [7]. The same LCM and FCS batches were used throughout the study.

To assess the frequency of macrophage precursors within the sorted subsets the macrophage colony- and clusterforming capacity of the sorted cells was determined in M-CSF-stimulated culture in soft agar. Cloning was performed as described by Wijffels et al. [8] with minor modifications. Briefly, a $6 \%$ agar stock (Bacto-Agar, Difco Laboratories, Detroit, MI) was diluted with warm $\left(42^{\circ} \mathrm{C}\right)$ culture medium. Cells were plated in $0.5 \mathrm{ml} 0.3 \%$ agar medium on top of a layer of $0.3 \mathrm{ml} 0.5 \%$ agar medium in 24-well plates (Costar, Cambridge, MA). Per sorted bone marrow subset two different cell concentrations were plated into six wells/concentration: 1000 and 250 cells/well for the ER-MP12 ${ }^{\text {med }} 20^{-}$, ER-MP12-20- , ER-MP12-20 $^{\text {med }}$ subsets and unlabeled, unseparated bone marrow, and 250 and 100 cells/well for the ER-MP12 ${ }^{\text {hi }} 20^{-}$, ER-MP12 $20^{+}$, and ER-MP12-20 ${ }^{\text {hi }}$ subsets. After 12 to 14 days of culture $\left(37^{\circ} \mathrm{C}, 7 \% \mathrm{CO}_{2}\right)$, the number of macrophage colonies ( $\geq 50$ cells) and clusters ( $<50$ cells) generated from each subset was assessed by examining the plates using an inverted light microscope at low magnification. LCM as a source of M-CSF specifically stimulates macrophage development, as only macrophage progeny could be detected using morphological and immunohistological analysis (data not shown).

For phenotypic analysis of in vitro matured mononuclear phagocytes, sorted cells were cultured in Teflon culture bags in M-CSF-containing medium [9]. Depending on the duration of culture, the initial cell number was adjusted to ensure optimal growth and viability. Thus, $2 \times 10^{4}$ cells were seeded in a volume of $2 \mathrm{ml}$ medium for phenotypic analysis at day 2 of culture, and $10^{4}$ cells, also in a volume of $2 \mathrm{ml}$, for analysis after 5 and 7 days of culture.

\subsection{Morphological analysis}

Differential morphological analysis of sorted bone marrow subsets was performed on May-Grünwald-Giemsa stained cytospin preparations. Per subset 500 cells were analyzed.

\section{Results}

\subsection{ER-MP12 and ER-MP20 mAb recognize morphologically distinct bone marrow subsets}

Using the anti-macrophage precursor $\mathrm{mAb}$ ER-MP12 and ER-MP20, six phenotypically distinct subsets can be detected in the bone marrow of the mouse [6]. Two subsets express the ER-MP12 antigen but not the ER-MP20 antigen: an ER-MP12 ${ }^{\text {med }} 20^{-}$and an ER-MP12 $22^{\text {hi }} 0^{-}$subset (Fig. 1). Similarly, two subsets exclusively express the ER-MP20 antigen: an ER-MP12-20 med and an ERMP12-20 ${ }^{\text {hi }}$ subset. Of the remaining two subsets, one expresses both ER-MP12 and ER-MP20 antigens, i.e. ER-MP12 ${ }^{+} 2^{+}$(for this subset no distinction was made in levels of antigen expression), and one lacks both antigens, 


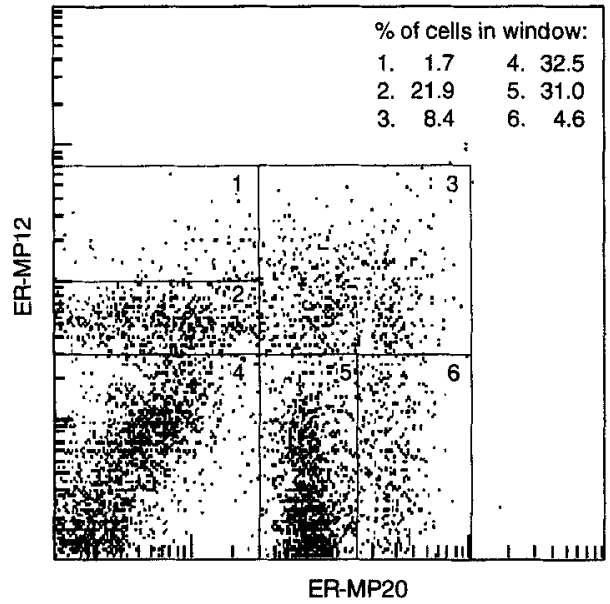

Figure 1. Two-color FACScan analysis of nucleated bone marrow cells labeled with ER-MP12 and ER-MP20 mAb. Percentages are the mean of eight experiments.

i.e. ER-MP12-20- . In this study we determined the cellular composition of the six ER-MP12/20 bone marrow subsets. Therefore each subset was sorted, stained with MayGrünwald-Giemsa and differentially counted (Table 1 and Fig. 2). The smallest subset, ER-MP12 ${ }^{\text {hi }} 20^{-}$, consisted predominantly of blast cells: morphologically undifferentiated blasts as well as recognizable blasts of the myeloid, erythroid and lymphoid lineages. The ER-MP12 $2^{\text {med }} 20^{-}$ subset was remarkably homogenous with predominantly mature lymphoid cells and a few undifferentiated blasts. The ER-MP12 $+20^{+}$subset contained a large proportion of morphologically undifferentiated blasts, together with recognizable precursors of the myeloid, erythroid and lymphoid lineages. The ER-MP12- $20^{-}$subset consisted almost exclusively of erythroid cells, erythroblasts as well as more mature cells. The ER-MP12-20 ${ }^{\text {med }}$ subset was highly enriched for granulocytes. Finally, the ER-MP12-20 hi subset contained mainly monocytes and a few immature myeloid cells and undifferentiated blasts. Thus, on the basis of ER-MP12 and ER-MP20 antigen expression, mouse bone marrow can be separated into six morphologically distinct subsets with a relatively high cell type homogeneity.

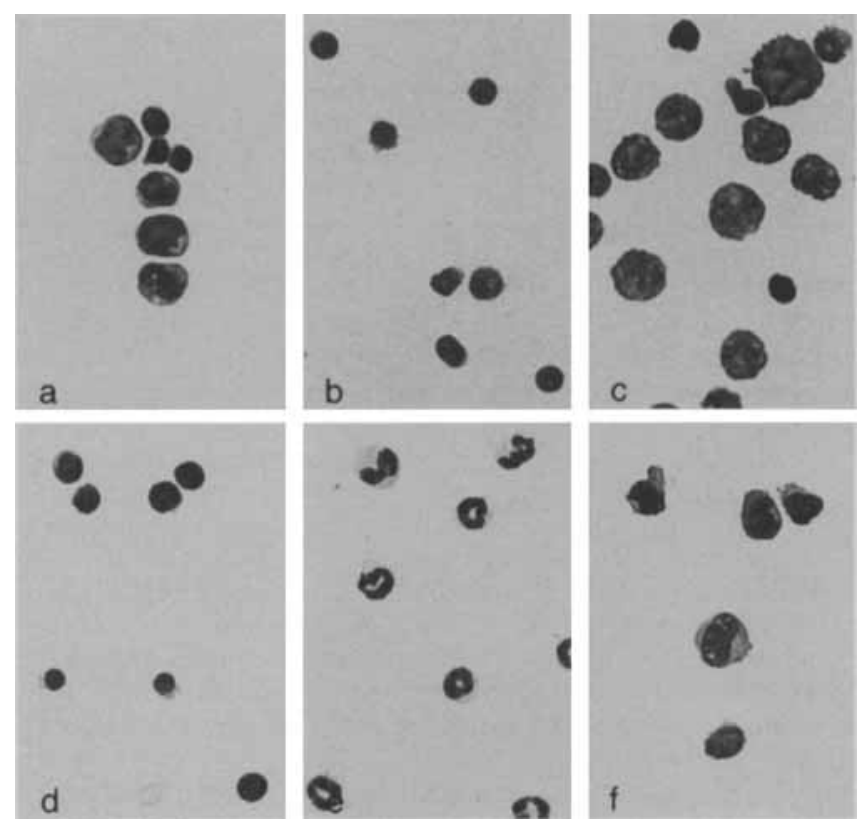

Figure 2. May-Grünwald-Giemsa-stained cytocentrifuge preparations of bone marrow subsets sorted on the basis of ER-MP12 and ER-MP20 expression. (a) ER-MP12 $20^{\text {hi }}$ - bone marrow subset; (b) ER-MP12med20- subset; (c) ER-MP12 ${ }^{+} 20^{+}$subset; (d) ERMP12-20- subset; (e) ER-MP12-20 med subset; (f) ER-MP12-20 ${ }^{\text {hi }}$ subset. Magnification $\times 350$.

\subsection{M-CSF-responsive macrophage precursors have the

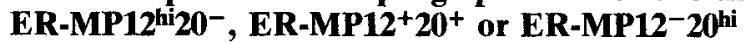 phenotype}

The morphological analysis of the sorted subsets showed that putative macrophage precursors, i.e. morphologically undifferentiated blasts, immature myeloid cells and monocytes, are mainly present in the ER-MP12 ${ }^{\text {hi20- }} 20^{-}$, ERMP12 ${ }^{\text {med }} 20^{-}$, ER-MP12 $20^{+}$, and ER-MP12- $20^{\text {hi }}$ subsets. To verify the presence of macrophage precursors in one or more of these subsets, and their absence from the other ER-MP12/20 subsets, all six bone marrow subsets were isolated by cell sorting and cloned in soft agar in the presence of the macrophage-specific growth factor M-CSF. Only from the ER-MP12 $22^{\text {hi }} 20^{-}$, ER-MP12 $20^{+}$and ER-

Table 1. Morphological analysis of the bone marrow subsets sorted on the basis of ER-MP12 and ER-MP20 antigen expression ${ }^{\text {a) }}$

\begin{tabular}{|c|c|c|c|c|c|c|c|c|}
\hline \multirow{2}{*}{$\begin{array}{l}\text { Bone marrow } \\
\text { subset }\end{array}$} & \multicolumn{3}{|c|}{ Myeloid } & \multicolumn{2}{|c|}{ Erythroid } & \multirow[t]{2}{*}{ Lymphoid } & \multirow{2}{*}{$\begin{array}{l}\text { Megakaryo- } \\
\text { cytic }\end{array}$} & \multirow{2}{*}{$\begin{array}{c}\text { Undifferentiated } \\
\text { blasts }\end{array}$} \\
\hline & $\begin{array}{c}\text { Immature } \\
\text { progenitors }\end{array}$ & $\begin{array}{c}\text { Band }+ \\
\text { segmented }\end{array}$ & Monocytes & Erythroblast & $\begin{array}{c}\text { Polychrom }+ \\
\text { normoblasts }\end{array}$ & & & \\
\hline ER-MP12hi $20^{-}$ & $4^{b)}$ & 0 & 1 & 18 & 3 & 25 & 0 & 49 \\
\hline ER-MP12 ${ }^{\text {med }} 20^{-}$ & 0 & 0 & 1 & 1 & 0 & 87 & 1 & $\frac{7}{10}$ \\
\hline ER-MP12+20+ & 20 & 7 & 7 & 14 & 3 & $\frac{7}{4}$ & 0 & 45 \\
\hline ER-MP12-20- & 0 & 0 & 0 & 15 & 76 & 0 & 0 & 9 \\
\hline ER-MP12 $20^{\text {med }}$ & 4 & 91 & 2 & 0 & 0 & 0 & 0 & 3 \\
\hline ER-MP12-20 $0^{\mathrm{hi}}$ & 19 & $\overline{0}$ & 74 & 1 & 0 & 0 & 0 & 6 \\
\hline
\end{tabular}

a) The sorted bone marrow subsets were spun onto microscopic slides and stained with May-Grünwald-Giemsa. Per subset 500 nucleated cells were examined.

b) Data represent the percentage of cells present in the ER-MP12/20 subsets. FACScan analysis of the sorted bone marrow subsets revealed that the purity of the sorted fractions varied between $87 \%$ for the ER-MP12+20+ subset and $>95 \%$ for the other subsets. In each subset the prevailing cell type is underlined. 
Table 2. Presence of M-CSF-responsive macrophage precursors in bone marrow subsets sorted on the basis of ER-MP12 and ER-MP20 antigen expression ${ }^{\mathrm{a}}$ )

\begin{tabular}{|c|c|c|c|c|}
\hline Bone marrow subset & $\begin{array}{l}\text { No. of colonies } / 10^{3} \\
\text { plated cells }{ }^{\mathrm{b})}\end{array}$ & $\begin{array}{l}\text { No. of clusters } / 10^{3} \\
\text { plated cells }\end{array}$ & $\begin{array}{c}\text { Mean frequency of macro- } \\
\text { phage precursors within } \\
\text { subset }\end{array}$ & $\begin{array}{c}\text { Mean no. of macrophage } \\
\text { precursors } 10^{4} \text { NBMC } \\
(\mathrm{M} \text {-CFC/M-clustFC) })^{\mathrm{c})}\end{array}$ \\
\hline ER-MP12hi20- & $108 \pm 9$ & $12 \pm 1$ & $1: 8$ & $20(18 / 2)$ \\
\hline ER-MP12+20+ & $22 \pm 8$ & $94 \pm 10$ & 1: 9 & $91(17 / 74)$ \\
\hline ER-MP12-20 & $0 \pm 0$ & $409 \pm 95$ & 1: 2 & $188(0 / 188)$ \\
\hline Unfractionated $\mathbf{B M}^{\mathrm{d})}$ & $4 \pm 1$ & $13 \pm 3$ & $1: 59$ & $170(36 / 134)$ \\
\hline
\end{tabular}

a) Macrophage colonies and clusters were determined after 12-14 days of M-CSF-stimulated culture in soft agar. Colonies contain $\geq 50$ cells; clusters $<50$ cells. No macrophage colonies or clusters were obtained from ER-MP12 $2^{\text {med }} 20^{-}$, ER-MP12- $20^{-}$, and ER-MP12-20med bone marrow subsets.

b) Data are the mean \pm SD of three experiments. For each subset, two different concentrations of sorted cells were plated. Per concentration the number of colonies and clusters per $10^{3}$ cells was calculated. The numbers shown in the table are the means of those calculated numbers. The numbers were corrected for the impurity of the sorted subsets; this was possible since contaminating cells were only from the subsets from which no macrophage colonies or clusters could be obtained. The means of the relative sizes of the bone marrow subsets in these three experiments were used to calculate the absolute numbers of macrophage precursors, and were $1.7 \%$ for the ER-MP12hi $20^{-}$subset, $7.9 \%$ for the ER-MP12 ${ }^{+} 20^{+}$subset and $4.6 \%$ for the ER-MP12- $20^{\text {hi }}$ subset.

c) NBMC: Nucleated bone marrow cells M-clustFC: Macrophage cluster-forming cell.

d) BM: Bone marrow.

MP12 $20^{\text {hi }}$ subsets could macrophage colonies and clusters be grown (Table 2). No macrophage colonies or clusters were obtained from the other three subsets.

In the ER-MP12 $122^{-}$subset one out of eight plated cells gave rise to primarily large macrophage colonies in response to M-CSF (Table 2), while in the ER-MP12+20+ subset one out of nine plated cells gave rise to primarily clusters and small colonies. In the ER-MP12-20 $20^{\text {hi }}$ bone marrow subset, which consists predominantly of monocytes (cf. Table 1), an average of one out of two plated cells formed a small cluster of usually less than 5 cells, and occasionally a cluster of $15-20$ cells. Taking into account the relative sizes of the sorted subsets in bone marrow, these data indicate that macrophage colony-forming cells reside in approximately equal numbers in both the ER-MP12 $120^{-}$ and ER-MP12 $20^{+}$subsets. Macrophage cluster-forming cells are mainly present in the ER-MP $12+20^{+}$and ERMP12 $-20^{\text {hi }}$ bone marrow subsets. Together, our data show that (i) M-CSF-responsive macrophage precursors reside in the ER-MP12 ${ }^{\text {hi }} 20^{-}$, ER-MP12 ${ }^{+} 20^{+}$, and ER-MP12-20 $20^{\text {hi }}$ subsets and (ii) the proliferative potential of the $\mathrm{M}$ CSF-responsive cells, as indicated by the sizes of the colonies or clusters formed, decreases with a concomitant increase in ER-MP20 and decrease in ER-MP12 Ag expression.

\subsection{The ER-MP12 $2^{\text {hi } 20^{-}}$, ER-MP12 ${ }^{+} 0^{+}$and ER-MP12-20 $0^{\text {hi }}$ bone marrow subsets differentially express the Mac-1 Ag}

The differences in cellular composition and proliferative potential of the ER-MP12 $120^{-}$, ER-MP12 $20^{+}$and ERMP12 $20^{\text {hi }}$ bone marrow subsets suggested a difference in maturation stage between the macrophage precursors present in those subsets. To investigate this, we examined the expression of the macrophage maturation-related marker Mac-1 $[10,11]$ within the three subsets. As shown in Fig. 3, all ER-MP12 ${ }^{\text {hi } 20^{-}}$cells were Mac-1-negative/dull. In contrast, $40 \pm 5 \%(n=3)$ of the ER-MP $12+20^{+}$bone marrow cells clearly expressed the Mac-1 Ag. This percent- age correlates closely with the total content of myeloid cells in this subset as determined by the morphological analysis (Table 1). Finally, virtually all ER-MP12-20 $0^{\mathrm{hi}}$ cells were Mac-1-positive. This observation was in accordance with the morphological data which showed that this subset almost exclusively contains monocytes and some immature myeloid precursors, which are all Mac-1-positive [10, 12]. Thus, the increase in the proportion of Mac-1 positive cells and level of $\mathrm{Ag}$ expression observed from the ERMP12 $120^{-}$to the ER-MP12-20 hi subset supports an increase in maturity of the macrophage precursors present in those subsets.

\subsection{ER-MP12 ${ }^{\text {hi } 20^{-}}$macrophage precursors successively express the ER-MP12 ${ }^{+} 0^{+}$and ER-MP12-20 ${ }^{\text {hi }}$ phenotype during M-CSF-stimulated maturation in vitro}

So far, the data obtained in this study suggest that the three macrophage precursor subsets represent successive stages in a linear maturation pathway, implying that the progeny of the putatively least mature subset, i.e. ER-MP12 $2^{\text {hi } 20^{-}}$, should pass through the other two phenotypes upon

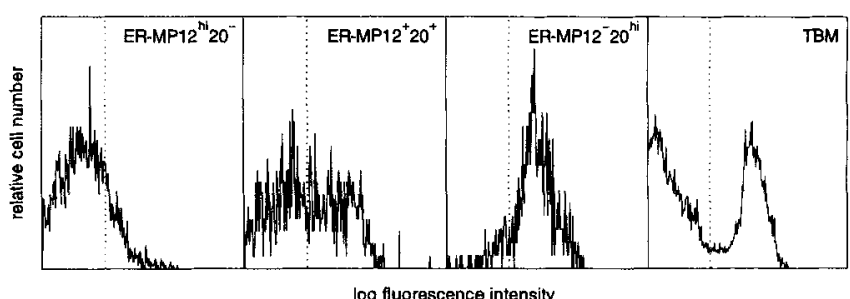

Figure 3. Cell surface expression of Mac-1 Ag by ER-MP12 $22^{\text {hi }}$ ER-MP12 $20^{+}$, and ER-MP12-20 $0^{\text {hi }}$ nucleated bone marrow cells Cells were triple-labeled as described in Sect. 2.4 with M1/70 (Mac-1), ER-MP12 and ER-MP20 mAb. Mac-1 expression within the ER-MP12/20 subsets was determined by flow-cytometric analysis. The staining profile of total bone marrow (TBM) is also given. The dotted lines represent negative control values. Results are from one representative experiment out of three. 
macrophage maturation. To test this hypothesis directly, we sorted ER-MP12 ${ }^{\text {hi }} 20^{-}$bone marrow cells and cultured the isolated cells in M-CSF-containing conditioned medium. After varying periods of culture the level of ER-MP12 and ER-MP20 Ag expression was determined. As a marker for mature macrophages, the expression of the ER-BMDM1 $\mathrm{Ag}$ was also examined. This latter antigen is expressed at increasing levels upon maturation from the monocytic stage onwards [5].

At day 2 of M-CSF-stimulated culture of ER-MP12hi20cells, about one third of the developing cells expressed both the ER-MP12 and the ER-MP20 antigens (Fig. 4A), suggesting that many, if not all, mononuclear phagocytes indeed pass through the ER-MP12+20+ $0^{+}$stage upon in vitro maturation. The remaining ER-MP12 ${ }^{+} 20^{-}$cells probably represented M-CSF-unresponsive cells from other hemopoietic lineages still present at this time of culture. At day 5 of culture, the cells had completely lost ER-MP12 antigen expression (Fig. 4A). Yet, $50-59 \%(n=2)$ of the cells expressed the ER-MP20 antigen at a high level, similar to that of ER-MP12-20 $0^{\text {hi }}$ monocytes in freshly isolated bone marrow (cf. Fig. 1). The ER-MP20-negative cells present after 5 days of culture represented mononuclear phagocytes beyond the monocytic stage of development as (i) at this day of culture $26-34 \%$ of the cells expressed the mature macrophage marker ER-BMDM1 (Fig. 4B) and (ii) the ER-MP20-negative cells showed a high level of autofluorescence (Fig. 4A, days 5 and 7) which is characteristic of more mature mononuclear phagocytes ([11] and unpublished observations). Upon final maturation into mature macrophages (day 7) the expression of the ER-MP20 antigen was lost (Fig. 4A) and the large majority of the cells (66-75\%) had become ER-BMDM1-positive (Fig. 4B).

In summary, these findings show that ER-MP12 2 hi20macrophage precursors successively express the ER-
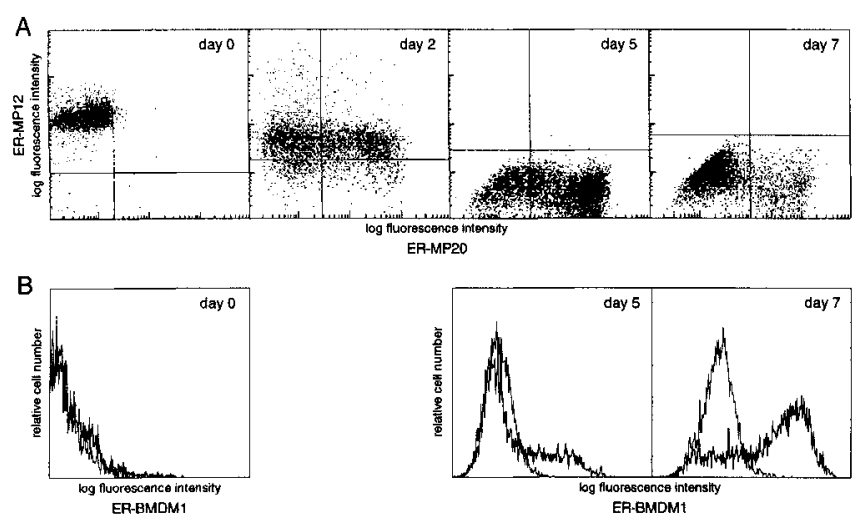

Figure 4. Phenotypic development of ER-MP12 $22^{-}$bone marrow cells cultured in the presence of M-CSF. ER-MP12 $22^{\text {hi }} 20^{-}$bone marrow cells were sorted and cultured in the presence of $M$ CSF-containing conditioned medium. At days 2,5 and 7 of culture, the cells were collected and the expression of ER-MP12 and ER-MP20 antigens was determined in two-color flow-cytometric analysis (A). Negative control limits are shown in each dot plot (vertical and horizontal lines). In addition, the expression of the macrophage maturation marker ER-BMDM1 was determined in one-color analysis (B). Negative controls (thin lines) are shown in each histogram. Results are from one representative experiment out of two.
$\mathrm{MP} 12^{+} 20^{+}$and ER-MP12-20 CSF-stimulated maturation in vitro. Under these conditions, ER-MP12-20- cells represent the final stage of macrophage development. The latter cells, however, are mature macrophages and do not represent the ERMP12- $20^{-}$subset found in normal mouse bone marrow.

\section{Discussion}

In the present study we focused on the identification of different subpopulations of macrophage precursors in the bone marrow of the mouse. To this end, the reactivity of the anti-macrophage precursor mAb ER-MP12 and ER-MP20 with bone marrow macrophage precursors was assessed in two-color flow cytometry. At least six phenotypically distinct bone marrow subsets can be discerned using these $\mathrm{mAb}$. However, only three of these subsets, i.e. ERMP12 $2^{\text {hi }} 20^{-}$, ER-MP12 $2^{+} 20^{+}$, and ER-MP12-20 ${ }^{\text {hi }}$, gave rise to macrophage progeny after M-CSF-stimulated culture. Together these subsets comprise about $15 \%$ of nucleated bone marrow cells.

All data presented in this study support the notion that these precursors reflect different, successive stages of macrophage development in the murine bone marrow. The first indication for the existence of a maturational difference came from morphological analysis of the sorted subsets. The differential counts showed that potential macrophage precursor cell types, i.e. morphologically undifferentiated blasts, immature myeloid cells, and monocytes, were not evenly distributed among the ER-MP12 $2^{\text {hi }}$ $20^{-}$, ER-MP12+20+, and ER-MP12- $20^{\text {hi }}$ subsets. Morphologically undifferentiated blasts were concentrated in the ER-MP12 $2^{\text {hi }} 20^{-}$and ER-MP12 ${ }^{+} 20^{+}$subsets, immature myeloid cells in the ER-MP12 $+20^{+}$and ER-MP12-20 hi subsets, whereas monocytes were concentrated in the ER-MP12- $20^{\mathrm{hi}}$ subset, thus suggesting differences in maturation stage among these subsets. A second indication was obtained from the differences in macrophage colony/cluster-forming capacities of the subsets. ER-MP12hizo- cells formed the largest colonies and therefore are presumably the most immature cells. ER-MP12 $20^{+}$and ERMP12- $20^{\text {hi }}$ cells formed predominantly large and small clusters, respectively, and thus, most likely represent subsequent stages. In the third place, the expression of the Mac-1 Ag, which is expressed relatively late during macrophage maturation $[10,11]$, follows the proposed maturation sequence of the precursor subsets. The Mac- $1 \mathrm{Ag}$ is not expressed in the ER-MP12 12 i $20^{-}$subset, while about half of the ER-MP12 ${ }^{+} 20^{+}$subset and all cells in the ER-MP12- $20^{\text {hi }}$ subset are Mac-1-positive. Finally, the most direct indication for the existence of a maturation sequence came from the phenotypic development of the, putatively youngest, ER-MP12 $2^{\text {hi }} 20^{-}$precursors during M-CSF-stimulated culture. We found that the developing cells successively passed through ER-MP12 $20^{+}$and ER-MP12-20 $0^{\text {hi }}$ stages before final maturation into mature macrophages. Thus, the morphological data, the clonogenic data, the expression of Mac-1 and the phenotypic development during culture all indicate that the three phenotypically distinct M-CSFresponsive bone marrow macrophage precursor subsets most likely represent successive stages along a maturation pathway in the order ER-MP12 ${ }^{\text {hi }} 20^{-} \rightarrow$ ER $\mathrm{MP} 12^{+} 20^{+} \rightarrow$ ER-MP12-20 ${ }^{\mathrm{hi}}$. 
Interestingly, macrophage colonies could be generated from both the ER-MP12 $20^{-}$and ER-MP12+20 ${ }^{+}$bone marrow subsets. Expressed in absolute numbers, about half of all bone marrow macrophage colony-forming cells resided in the ER-MP12 ${ }^{\text {hi }} 20^{-}$subset and the other half in the ER-MP12 ${ }^{+} 20^{+}$subset. MacVittie [13] reported for C57BL/6J mice an M-CFC frequency in bone marrow of approximately twice the frequency of GM-CFC.Thus, of all macrophage colonies formed in their study, about two thirds was derived from an M-CFC and one third from a GM-CFC. Combining these data with our observations on ER-MP12/20 expression by macrophage colony-forming cells, we speculate that the ER-MP12 $2{ }^{\text {hi }} 20^{-}$macrophage precursors, which form the largest colonies, represent the more immature GM-CFC and part of the M-CFC, while the colony-forming macrophage precursors in the ER$\mathrm{MP} 12^{+} 20^{+}$subset might represent the majority of the $\mathrm{M}-\mathrm{CFC}$.

Our data showed that the earliest $\mathrm{M}-\mathrm{CFC}$ in the bone marrow are ER-MP12 $2{ }^{\text {hi }}{ }^{-}$. Recently splenic M-CFC were found to express the ER-MP20 Ag at a high level and thus differ from bone marrow M-CFC, which are ER-MP20- or ER-MP20 dim [14]. It is unlikely that this difference in ER-MP20 expression reflects a maturational difference since the splenic ER-MP20 $0^{\text {hi }}$ cells are, like the bone marrow ER-MP20- and ER-MP20 dim cells, able to form large macrophage colonies in culture. Therefore, it will be interesting to study the ER-MP12 Ag expression of splenic $\mathrm{M}-\mathrm{CFC}$, as, in bone marrow, this is clearly related to colony-forming capacity.

Both the differential counts and the macrophage precursor frequencies (cf. Tables 1 and 2) of the ER-MP12 $12^{\text {hi }} 20^{-}$and ER-MP12 ${ }^{+} 20^{+}$subsets indicate that these fractions do not solely contain precursors of the macrophage lineage. Morphological analysis of the subsets showed that precursors of the erythroid, lymphoid and granulocytic lineages are present in these subsets. In accordance with this observation, we recently reported that ER-MP12 ${ }^{\text {hi }} 20^{-}$bone marrow cells gave rise to both myeloid cells as well as Tand $B$ cells upon intravenous (i.v.) transfer into irradiated recipients [15]. Thus, although the ER-MP12hi $20^{-}$and ER-MP12+ $20^{+}$bone marrow subsets are both highly enriched for macrophage precursors, additional cell surface markers are required to separate early macrophage precursors from other hemopoietic progenitors.

A remarkable finding was that no macrophage colonies or clusters could be grown from ER-MP12 $12^{\text {med }} 20^{-}$bone marrow cells in M-CSF-stimulated culture, although we recently reported that ER-MP12 ${ }^{\text {med }} 20^{-}$bone marrow cells, upon i.v. transfer into irradiated recipients, were capable of both myeloid and $\mathrm{T}$ and $\mathrm{B}$ cell repopulation [15]. This apparent contradiction can be explained by the presence of more immature progenitors and multipotent stem cells within the ER-MP12 ${ }^{\text {med }} 20^{-}$subset (J.C.M van der Loo et al., manuscript in preparation). Such cells do not yet respond in culture to M-CSF alone. In vivo, however, the cells most likely meet the appropriate microenvironments and will eventually become sensitive to M-CSF and form mature macrophage progeny.

To our knowledge, ER-MP12 and ER-MP20 are the first set of $\mathrm{mAb}$ described which positively identify discrete, successive macrophage precursor stages in the bone marrow of the mouse. Furthermore, as the ER-MP12 ${ }^{\text {hi }} 20^{-}$bone marrow subset was found to contain macrophage precursors as well as precursors of other lineages, it may be possible, using additional markers, to identify early branch points in hemopoiesis.

We would like to thank Mr. J. Brandenburg and Ms. E. van Bodegom for animal care, $M r . T . M$. van Os for preparation of the figures and Dr. R. E. Ploemacher for his continuous support.

Received June 10, 1994; accepted June 27, 1994.

\section{References}

1 Leenen, P. J. M. and Campbell, P. A., in Horton, M. A. (Ed.), Blood Cell Biochemistry. Vol. 5, Macrophages and Related Cells, Plenum Press, New York 1993, p.29.

2 Rutherford, M. S., Witsell, A. and Schook, L. B., J. Leukoc. Biol. 1993. 53: 602 .

3 Leenen, P. J. M., Melis, M., Slieker, W. A. T. and van Ewijk, W., Eur. J. Immunol. 1990. 20: 27.

4 Springer, T., Galfré, G., Secher, D. S. and Milstein, C., Eur. J. Immunol. 1979. 9: 301.

5 Leenen, P. J. M., Melis, M., Kraal, G., Hoogeveen, A. T. and van Ewijk, W., Eur. J. Immunol. 1992. 22: 1567.

6 Slieker, W. A. T., de Rijk-de Bruijn, M. F. T. R., Leenen, P. J. M. and van Ewijk, W., Int. Immunol. 1993. 5: 1093.

7 Leenen, P. J. M., Slieker, W. A. T., Melis, M. and van Ewijk, W., Eur. J. Immunol. 1990. 20: 15.

8 Wijffels, J. F. A. M., de Rover, Z., Kraal, G. and Beelen, R. H. J., J. Leukoc. Biol. 1993. 53: 249.

9 van der Meer, J. W. M., van de Gevel, J. S., Elzenga-Claassen, I. and van Furth, R., Cell. Immunol. 1979. 42: 208.

10 Miller, B. A., Antognetti, G. and Springer, T. A., I. Immunol. 1985. 134: 3286.

11 Walker, E. B., Akporiaye, E. T., Warner, N. L. and Stewart, C. C., J. Leukoc. Biol. 1985. 37: 121.

12 Nibbering, P. H., Leijh, P. C. J. and van Furth, R., Cell. Immunol. 1987. 105: 374.

13 MacVittie, T. J., Bibl. Haemat. 1984. 48: 112.

14 McCormack, J. M., Leenen, P. J. M. and Walker, W. S., J. Immunol. 1993. 151: 6389.

15 Slieker, W. A. T., van der Loo, J. C. M., de Rijk-de Bruijn, M. F. T. R., Godfrey, D. I., Leenen, P. J. M. and van Ewijk,W., Int. Immunol. 1993. 5: 1099. 